\title{
Streamlined engineering for synthetic biology
}

\author{
Jeffrey M Perkel \\ Researchers are injecting ever more conscious design into their bioengineering workflow.
}

Yogurt. Comedian George Carlin wouldn't touch it; Spaceballs villain "Dark Helmet" "hated" it-even with strawberries. But Boston University biomedical engineer James Collins, a Howard Hughes Medical Institute Investigator, has recently been giving the fermented dairy treat a great deal of thought. Not how his preferred flavor, vanilla, tastes atop granola, nor how plain yogurt and dill pair perfectly with salmon. For Collins, yogurt-or, more precisely, the lactobacilli that make yogurt yogurt-could potentially be the answer to a vexing public health problem: cholera.

Collins is a pioneer in the burgeoning field of synthetic biology, the science of reprogramming cells with novel biological functions, from biosensors to antimicrobials to biofuels. The field, writes Harvard Medical School geneticist George Church, capitalizes on the fact that biological organisms are effectively programmable manufacturing systems. In this context, cells serve as miniature computers, programmed toward a new purpose with externally supplied genetic instructions. Church notes that "making small changes in their genetic software a bioengineer can effect big changes in their output" .

In Collins's case, the big change he hopes to effect converts yogurt cultures into microbial sentries against Vibrio cholerae, the pathogen that causes cholera. Supported by a grant from the Bill and Melinda Gates Foundation, Collins wants to harness the pathogen's own biochemistry against it. His team is working to genetically reprogram yogurt bacteria to recognize the small molecules that the cholera bacteria use to communicate and sense their environment, in a process called "quorum sensing," and pump out anti-cholera peptides in response. "You've got basically now a little bug that could become your instant

pharmacy to respond to the infection," says Collins.

To do their protective job, the lactobacilli would be equipped with a synthetic circuit that recognizes two different inputs, one for each signal. Only upon receipt of both signals would the circuit switch on, adding a layer of protection against false positives. In the parlance of computer programming, such a circuit is said to comprise an AND logic gate, one in which two input conditions must be met before the gate opens, such that only receipt of both signals triggers the organism to fight the infection. "You could envision giving pills or spiked yogurt to people in the face of a cholera outbreak in the hope that this could strengthen them and/or give them a fighting chance against the infection," Collins explains. That approach is better than trying to dose everyone in the area with potentially toxic, and definitely expensive, antibacterials, he says.

The idea seems to be simple enough for a quick sketch: if $\mathrm{X}$ and $\mathrm{Y}$ molecules are in the gut, the person is infected-let's do something about that. Yet building a working circuit isn't as easy as it seems, most notably because biological systems are not binary but stochastic. A synthetic circuit requires that an OFF promoter really be

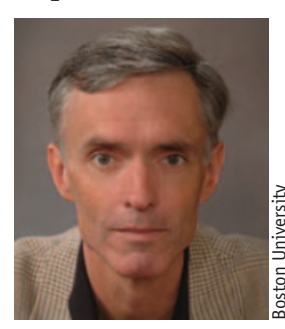

Jim Collins is converting yogurt cultures into microbial sentries against the pathogen that causes cholera. off, for instance, but genetic circuits are often leaky, producing transcripts even in the absence of an activating signal. "Biology is still very far removed from being an engineering discipline," Collins says. Still, he and others in the synthetic biology com-

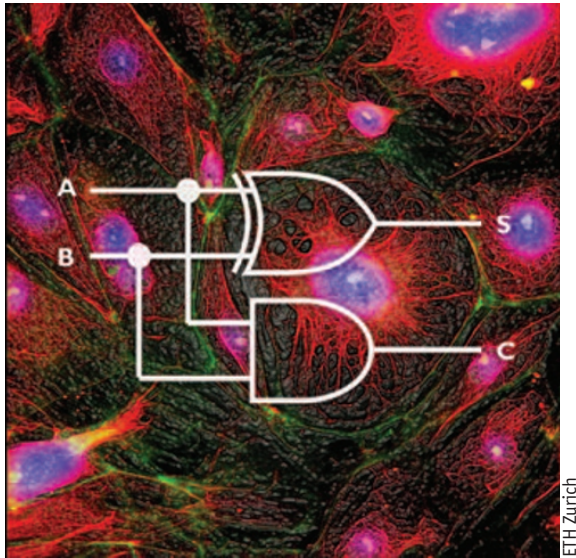

Systems biology applications include everything from total genome rewrites to simple pathway engineering.

munity are on the case. With advances in biological components, cellular communication and experimental design, researchers are injecting ever more engineering sensibility into the synthetic biology workflow. If things work out as hoped, biologists can look forward to a time when synthetic biology mimics electronic engineering, a time when the design of a biocircuit is as simple as drawing a picture.

\section{Cellular cross-talk}

Systems biology applications include everything from total genome rewrites to simple pathway engineering. For Martin Fussenegger, a bioengineer in the Department of Biosystems Science and Engineering at the Swiss Federal Institute of Technology (ETH) Zurich in Basel, synthetic biology is a useful tool for addressing biomedical therapeutics.

He builds what he calls "prosthetic networks," which are molecular circuits that combine a sensor and a therapeutic output to affect a condition in an animal disease model. The circuits are not unlike Collins's 


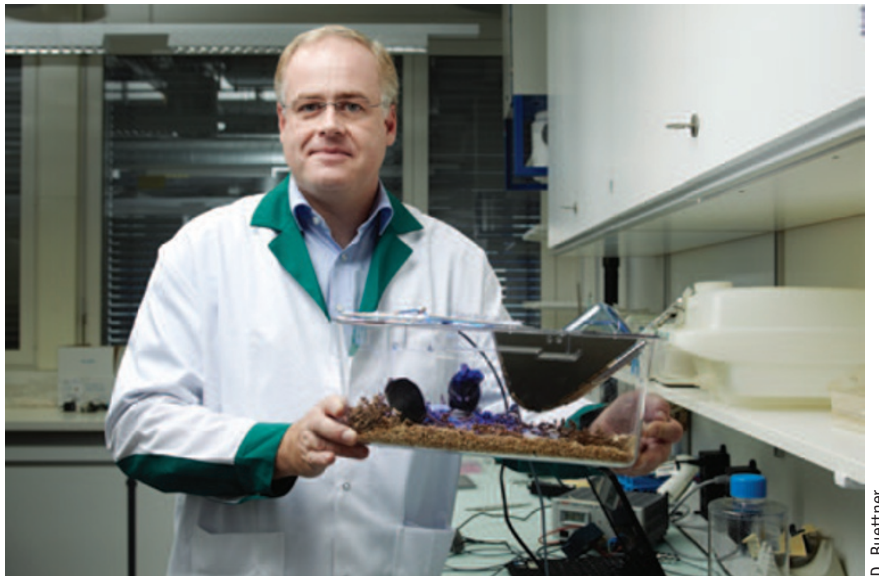

Martin Fussenegger's type 2 diabetic mice have cell implants that restore glucose homeostasis when the mice are illuminated with blue light.
1 (GLP1) to control glucose levels when activated with blue light.

Last July, his team announced its most advanced "biocomputer" yet. By combining simple "plug-and-play" transcription and translation control circuits in cultured human HEK-293 cells, they built a series of complex logic circuits from sophisticated build-

planned anti-cholera agent in design. Collins calls Fussenegger's approach the "best and most productive mammalian synthetic biology work" and says it has "real clinical relevance." In 2010, Fussenegger's team developed a prosthesis for gouty arthritis, a condition caused by excessive levels of uric acid, which crystallizes painfully in the joints and also causes kidney damage.

His solution is to endow cells with a sensory circuit, embed those cells in a porous implantable matrix such as alginate, a polymeric polysaccharide, and then implant the resulting composite into animals. In the animal model of gouty arthritis, a missing enzyme means that elimination of uric acid is no longer possible. The engineered circuit supplies that missing enzyme, plus DNAbinding proteins and regulatory sequences that induce its expression when uric acid levels rise. Fussenegger's team introduced those elements into HeLa cells and encapsulated them in alginate, creating a porous human cellular implant that responds to uric acid levels in mice. The alginate insulates the implanted cells from the animal's immune system, yet its pores permit the cells to sense and respond to the environment.

When the cells are implanted into the animal model of gouty arthritis, he says, the scientists observed physical changes: whereas the animals had walked with difficulty before, after a few days they appeared more mobile. "The crystals in the joints and mainly in the kidney start to be dissolved, and the animal gets back to near-normal moving capacity," he says ${ }^{2}$.

Fussenegger has developed several implementations of this basic formula, including a photoresponsive implant for type 2 diabetes that secretes glucagon-like peptide works can get, he says, at least if confined to single cells. "Mammalian cells have a life of their own, so they need to take care of their metabolism and of their life." In other words, genetic programming aside, engineered cells take care of themselves first and only pursue the engineer's goal in what he calls "their spare time."

To spread the workload between cells, Fussenegger has expanded into more conversational networks. He has begun developing multicellular systems in which circuits are divided among different cell types just as crowdsourced applications distribute work among many computers. His team built a circuit in which two cells carry on a molecular conversation. Cell A synthesizes tryptophan in response to exogenously added indole. That tryptophan signal is picked up by cell B; in response, cell B secretes acetaldehyde, which in turn activates expression of a target protein in cell $\mathrm{A}^{3}$.

"It's really two-way communication," Fussenegger says. "It's not just A talks to $B$, but it's a conversation of A constantly talking to B, and B talking back to A." That conversation, of course, is carried out with metabolites, and the particular language was chosen with purpose. "We didn't want to have artificial small-molecule drugs floating around in our system," he says. But the fact that both tryptophan and acetaldehyde are normal cellular metabolites did complicate
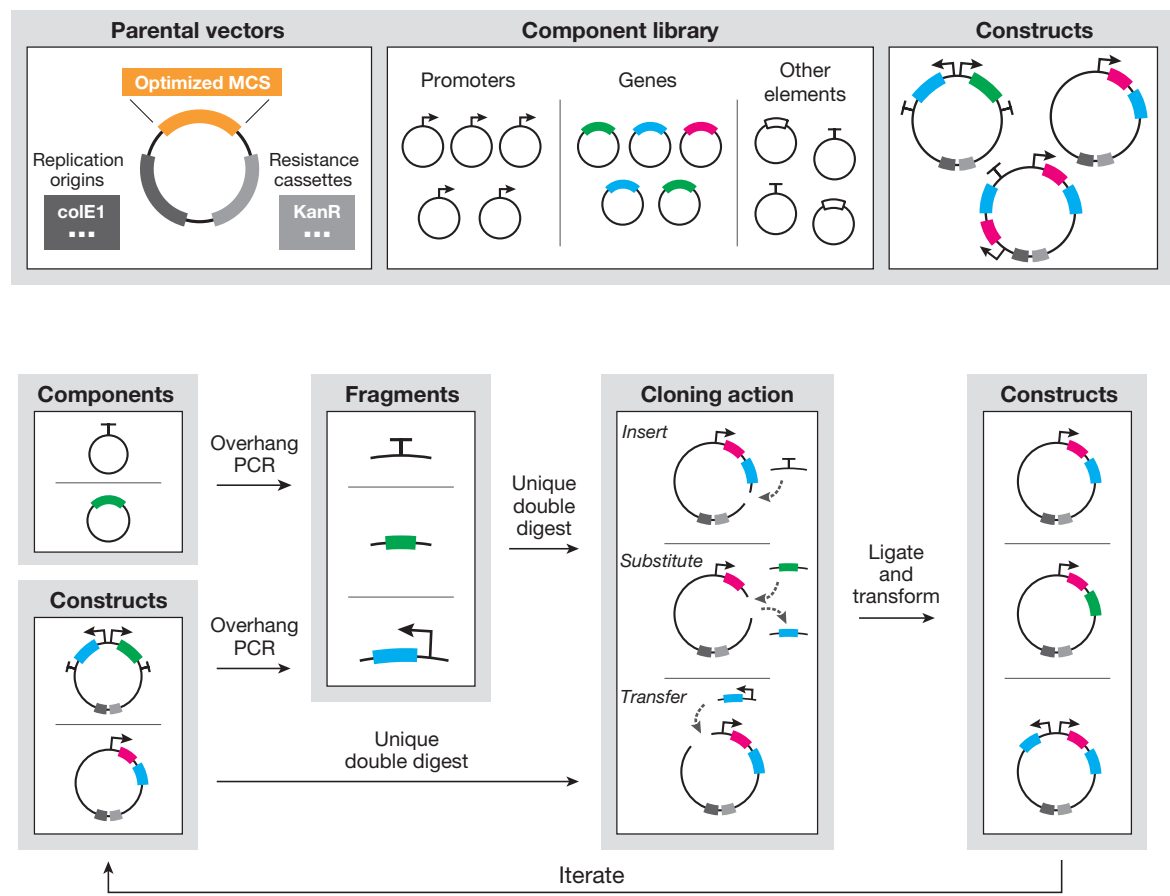

This synthetic biology 'breadboard' design allows Jim Collins's lab to rapidly prototype and optimize biological circuits. The fundamental components (top) are combined using an iterative workflow comprising PCR, restriction digestion and ligation (bottom). (Adapted from ref. 6.) 


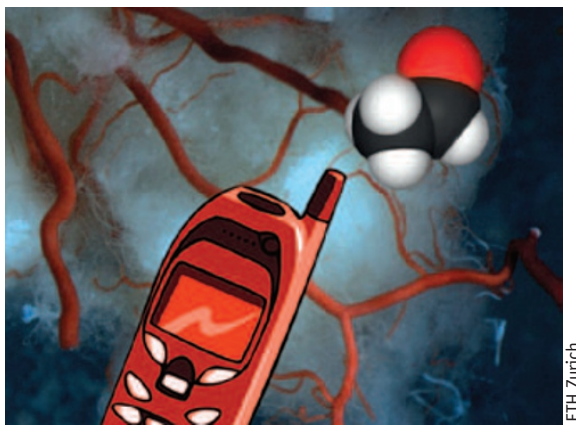

Martin Fussenegger's team has built a distributed circuit that is closed when two cells carry on a molecular conversation.

the system, he adds, because both molecules are used for normal cell operation, and only the excess can be used for communication.

Still, the circuit is about more than a couple of small molecules; those are just molecular semaphores, or signal flags. The team also endowed these cells with additional circuitry such that cell B also produces vascular endothelial growth factor (VEGF) in response to tryptophan, and cell A produces the antagonistic signal, angiopoietin, in response to acetaldehyde. Both cells were then cultured below a permeable membrane containing endothelial cells. As those endothelial cells responded first to VEGF, and later to angiopoietin, they showed altered permeability, which is a marker of angiogenesis.

Nature, Fussenegger says, has created many such systems, in which biological processes are controlled by mutually exclusive protein-control circuits. Now, using the circuit designs he and his team have developed, researchers can mimic and engineer those circuits in the lab.

\section{Standards for use and re-use}

At Stanford University, bioengineer Drew Endy is working on ways to make circuits from Fussenegger's lab and others reusable by creating standardized biological parts. Endy likens his project's goal to the challenge of building with rocks. Because rocks have different shapes and sizes, they cannot easily be used to build or repair a wall. Using rocks that are cut to a standard size and shape in a quarry, however, makes wall repair simpler.

Standardization, Endy says, "allows for coordination of labor across location and over time." Coordinating labor in this way, "you can enable human beings to work together and realize things that would be impossible for any one person to do alone."
For the past decade he has been involved in the BioBricks project, a community effort trying to do for biology what rock quarries do for builders: standardization, or squaring off the edges.

But BioBricks are about more than merely squaring edges, Endy says: "There's four different categories of technical standards that can be invoked to describe aspects of an object with the BioBrick label." These categories are physical assembly, or how pieces fit together; functional composition, or ensuring that the composite piece functions as expected; data exchange; and measurement.

Jason Kelly, a former Endy graduate student who went on to cofound a synthetic biology company, Gingko BioWorks, developed in Endy's lab a standard unit of measurement for expression activity, the "relative promoter unit." That, says Endy, allows researchers to be quantitative in describing biological components, rather than using qualitative descriptors like "good," "strong" or "high."

As Endy explains, Kelly declared a particular combination of promoter, ribosome binding site (RBS) and GFP, called BBa_J23101, to be "the equivalent of a meter stick." When researchers measure gene expression, his idea was to have them "make a parallel measurement against the meter stick and then use that to calibrate their local measurements"4. As with all useful meter sticks, this one is readily available; researchers can obtain it from partsregistry.org.

Endy is also addressing functional composition standardization, the idea that two pieces not only can be put together but will then work as intended. The poster child for

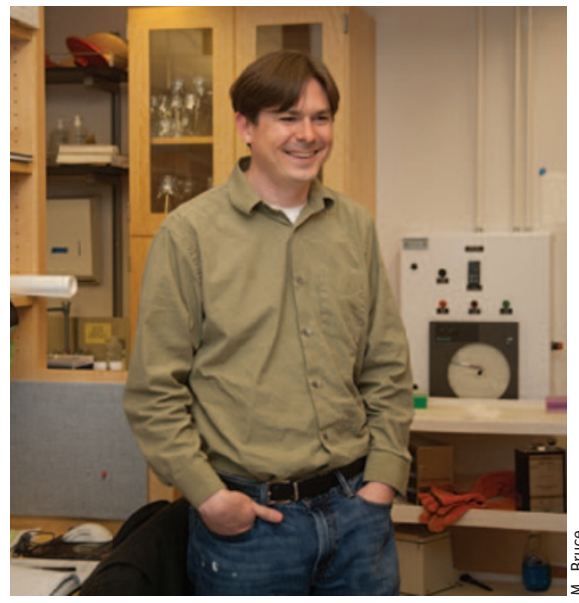

Christopher Voigt is tweaking biological components so that they behave as expected in synthetic systems. the idea, he says, is bacterial protein expression.

Bioengineers would like to be able to swap RBSs at will, for instance to increase or decrease protein expression levels fivefold. But an RBS that works well in one context may not work as well in another, which tends to lead to a lot of post-circuit design tweaking. "If I represent to you, 'Hey, I've got the world's best ribosome-binding site, it's really strong,' and you show up with your favorite gene, do we actually know whether or not it'll work? Will the composite object function as we expect?" The challenge is that RBS strength varies with secondary structure, which, in turn, is sequence dependent. Endy's team is working to address that problem.

Bioengineer Christopher Voigt, at the Massachusetts Institute of Technology, has tackled a related challenge: how to decouple promoter strength from translational efficiency, again in order to achieve component modularity. "When you switch promoters, you end up with different nucleotides at the $5^{\prime}$ end of the transcript, which can impact [protein] expression," he says.

Such seemingly trivial differences can have "really dramatic effects," Voigt says, creating situations in which a rational engineering exercise can turn into an evolutionary approach requiring many random changes. "You have to take a lot of shots on goal."

To circumvent that exercise, Voigt's team conducted a screen to identify sequence elements that could be inserted after the transcription start site to effectively standardize the $5^{\prime}$ untranslated region of an mRNA transcript, thereby decoupling translational efficiency from the promoter sequence ${ }^{5}$.

The screen identified a ribozyme sequence that, when inserted between a promoter and the transcription start site, cleaves the transcript's $5^{\prime}$ untranslated region at a specific spot. "So no matter how much [sequence] is tacked on from the promoter, it gets cleaved off at a specific site so that the transcript itself stays the same," he says.

Building on this finding, Voigt can now be reasonably sure that any difference he sees in protein abundance in a synthetic circuit is related to the promoter's activity and not to the particular sequence it tags onto the $5^{\prime}$ end of the transcript. He is now testing that idea using promoters of different strength for his work on nitrogen fixation pathways. "In some of the larger designs we are building, every single gene has its own ribozyme at this location," he says. 


\section{Accelerated circuit development}

Despite all of its advances, synthetic biology remains a highly empirical enterprise, says Collins. Synthetic biologists create circuits to match preconceived designs, then spend even more time optimizing those circuits to perform as intended. Now Collins and his team have devised a way to accelerate that process.

Collins and colleagues described an "iterative plug-and-play methodology for constructing and modifying synthetic gene networks" that is inspired by the electrical engineer's 'breadboard', a solderless template that allows for easily swapping out components on a circuit board ${ }^{6}$.

"This was a plasmid that was specifically designed [so] that you could pop in and out readily, say up to six different genes and the associated parts, to create [or] fast-track synthetic gene networks," he explains. Using a set of 26 plug-and-play plasmids, promoters, genes and control elements, and a suite of standard molecular procedures-polymerase chain reaction (PCR), restriction digestions and ligation reactions - the team was able to build and optimize a molecular toggle switch in just over a week. They then converted that circuit into a pair of feedforward loops, all by the same iterative PCRdigest-ligate strategy, in just five days.

"Instead of looking at many months, as is common in synthetic biology, we showed that in really a matter of days you can get...cool circuits that behave as desired," Collins says.

Such tools stand to aid Collins in his work to make a prophylactic yogurt. But the rest of the synthetic biology community will benefit, too. If, as Church writes, biological organisms are essentially "programmable manufacturing systems" driven by "genetic software," then Collins's design gives synthetic biologists a next-generation programming tool to ease development.

Bottom line: new synthetic biology apps are coming, faster than you think.

1. Church, G. \& Regis, E. Regenesis: How Synthetic Biology Will Reinvent Nature and Ourselves (Basic Books, New York, 2012).

2. Kemmer, C. et al. Nat. Biotechnol. 28, 355-360 (2010).

3. Bacchus, W. et al. Nat. Biotechnol. 30, 991-996 (2012).

4. Kelly, J.R. et al. J. Biol. Eng. 3, 4 (2009).

5. Lou, C. et al. Nat. Biotechnol. 30, 1137-1142 (2012).

6. Litcofsky, K.D. et al. Nat. Methods 9, 1077-1080 (2012).

Jeffrey M. Perkel is a freelance writer based in Pocatello, Idaho. 